\title{
Two-step method for the deposition of AlN by radio frequency sputtering
}

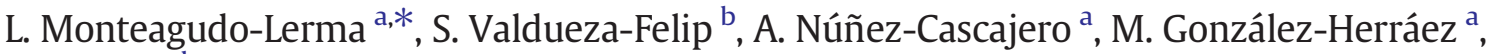 \\ E. Monroy ${ }^{\text {b }}$, F.B. Naranjo ${ }^{\text {a }}$ \\ a Grupo de Ingeniería Fotónica, Departamento de Electrónica (EPS) Universidad de Alcalá, Campus Universitario, 28871 Alcalá de Henares, Madrid, Spain \\ b CEA-Grenoble, INAC/SP2M/NPSC, 17 rue des Martyrs, 38054 Grenoble, France
}

\section{A R T I C L E I N F O}

\section{Article history:}

Received 2 August 2012

Received in revised form 19 July 2013

Accepted 23 July 2013

Available online 31 July 2013

\section{Keywords:}

Semiconductors

Aluminum nitride

Sputtering

Substrate bias

High resolution X-ray diffraction

Two-step method

\begin{abstract}
A B S T R A C T
This paper presents a detailed study of the influence of deposition conditions on structural and morphological properties of AlN thin films synthesized on $c$-sapphire substrates by radio frequency (RF) reactive sputtering. After the optimization of deposition parameters such as RF power and substrate temperature, the substrate bias has been identified as a critical variable to improve the structural properties of the AlN layers. The use of negative bias leads to a decrease of the full-width at half-maximum (FWHM) of the rocking curve of the AlN-(0002) $\mathrm{X}$-ray reflection and an increase of the grain size. However, $2 \theta / \omega \mathrm{X}$-ray scans of layers grown under negative bias reveal lattice disorder at the AlN/sapphire interface, which is attributed to the highly accelerated positive ions $\left(\mathrm{Al}^{+}, \mathrm{N}^{+}, \mathrm{N}_{2}^{+}\right)$arriving to the substrate at the initial stages of the deposition process. In order to prevent this interface degradation, we propose a two-step deposition method which consists of starting the growth with an unbiased AlN buffer layer, at least $30 \mathrm{~nm}$ thick, followed by AlN deposition under negative bias. This procedure results in high-quality AlN layers with FWHM of the rocking curve of the (0002) reflection of $1.63^{\circ}$, grain size of $\sim 40 \mathrm{~nm}$ and root-mean-square surface roughness of $0.4 \mathrm{~nm}$.
\end{abstract}

(C) 2013 Elsevier B.V. All rights reserved.

\section{Introduction}

AlN has become an attractive material due to its interesting combination of properties, like large direct band gap energy $(6.2 \mathrm{eV})$, high acoustic velocity, strong piezoelectricity and mechanical robustness [1]. All these features make AlN a suitable semiconductor for application in optical components in the ultraviolet spectral region [2,3], surface acoustic wave devices [4], micro-electro-mechanical systems [5] and coating layers. AlN is also widely used as buffer layer for the growth of $\mathrm{GaN}$, in order to reduce the lattice mismatch, stabilize the metal polarity and prevent unwanted reactions between GaN and the substrate [refs. 6-8].

The most common techniques used for AlN growth are metalorganic vapor phase epitaxy $[9,10]$, hydride vapor phase epitaxy [11] and molecular beam epitaxy $[12,13]$, all requiring expensive technology and high substrate temperature. Radio frequency (RF) reactive magnetron sputtering represents an attractive low-cost technique to synthesize AlN films, allowing deposition in a wide range of temperatures and in both rigid and flexible substrates [refs. 14-16].

In this work, we first present the optimization of the deposition parameters for the growth of $c$-AlN thin films on sapphire by RF reactive sputtering in pure nitrogen atmosphere. Then, we describe the effect of the substrate bias on the crystalline and morphological properties of the optimized layers. The obtained results are discussed in terms of

\footnotetext{
* Corresponding author. Tel.: + 3491885 6913; fax: + 34918856591.

E-mail address: laura.monteagudo@depeca.uah.es (L. Monteagudo-Lerma).
}

the energy of the impinging species reaching the substrate. Finally, we introduce a two-step deposition method which consists of the deposition of a first AlN thin buffer layer under unbiased substrate conditions (floating substrate, FS) followed by a negatively biased AlN layer.

\section{Experimental}

AlN samples were deposited on c-sapphire substrates in an RF sputtering system equipped with a 2-inch confocal magnetron cathode (AJA International, ATC ORION-3-HV). The growth proceeded in pure nitrogen atmosphere using a 2-inch Al target ( $5 \mathrm{~N}$ purity) and highpurity (6 N) $\mathrm{N}_{2}$ gas. The distance between target and substrate was fixed to $10.5 \mathrm{~cm}$, and the areas of the substrate holder and the substrate were kept constant to 81 and $7 \mathrm{~cm}^{2}$, respectively. A thermocouple placed in contact with the substrate holder allowed monitoring the substrate temperature during the deposition process. Prior to growth, the substrates were cleaned in organic solvents and outgassed in the deposition chamber for $30 \mathrm{~min}$ at a substrate temperature $100{ }^{\circ} \mathrm{C}$ higher than the growth temperature to ensure a base pressure for the growth of $\sim 10^{-5} \mathrm{~Pa}$. The Al target was cleaned with Ar plasma before each deposition in order to remove the contamination of target surface induced during the substrate loading process.

The structure of the AlN layers was investigated by high-resolution X-ray diffraction (HRXRD) with a Bruker AXS D8 Advance four-axis diffractometer using $\mathrm{Cu} \mathrm{K} \alpha_{1}$ incident radiation. The layer grain size was estimated from the full-width at half-maximum (FWHM) of the AlN-(0002) reflection peak of the $2 \theta / \omega$-scan by means of Scherrer's 
formula [17]. The surface roughness of the AlN layers was measured by atomic force microscopy (AFM) in the tapping mode with a Veeco Dimension 3100 microscope. Data visualization and processing were carried out using the WSxM software [18].

\section{Results and discussion}

\subsection{Deposition of AlN layers under unbiased substrate conditions}

The first step towards the optimization of AlN deposition consisted in studying the effect of deposition parameters such as RF power $\left(P_{\mathrm{RF}}\right)$ and substrate temperature $\left(T_{\text {subs }}\right)$ on the layer properties. Taking into account previous results [16], the sputtering pressure was fixed to $0.47 \mathrm{~Pa}$.

A set of AlN layers was deposited at $T_{\text {subs }}=400{ }^{\circ} \mathrm{C}$ with RF power ranging from $100 \mathrm{~W}$ to $175 \mathrm{~W}$. For all the samples of this set, the

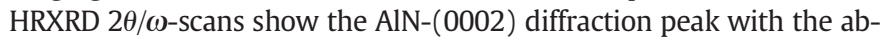
sence of reflections related to other crystal orientations. Fig. 1(a) shows the dependence of the FWHM of the $\omega$-scan around the (0002) AlN x-ray reflection peak on $P_{\mathrm{RF}}$. A decrease of this FWHM from $4.26^{\circ}$ to $2.18^{\circ}$ is observed when increasing $P_{\mathrm{RF}}$ from $100 \mathrm{~W}$ to $150 \mathrm{~W}$. This amelioration is attributed to the increase of both the Al species energy and the target sputtering rate, the latter resulting in an increase of the growth rate from $40 \pm 5 \mathrm{~nm} / \mathrm{h}$ to $60 \pm 5 \mathrm{~nm} / \mathrm{h}$ within that $P_{\mathrm{RF}}$ range. This improvement of crystal quality and growth rate with the RF power is in agreement with results presented by other authors [19]. The effect of sputtering gas rarefaction can be neglected considering the low deposition pressure and atom flux in our experiment $(0.47 \mathrm{~Pa}$ and $7.8 \times 10^{18}$ atoms $/ \mathrm{m}^{2} \mathrm{~s}$, respectively) [20,21]. Furthermore, the increase of the energy of the impinging species, and thus their mobility on the growing surface, leads to a decrease of the root-mean-square (RMS) roughness of the AlN layers from $1.8 \mathrm{~nm}$ to $0.3 \mathrm{~nm}$ [see Fig. 1(a)]. Further increase of $P_{\mathrm{RF}}$ up to $175 \mathrm{~W}$ leads to a degradation of the crystalline quality and surface morphology of the layer, with values of FWHM of the AlN-(0002) related rocking curve of $3.80^{\circ}$ and RMS roughness of $0.6 \mathrm{~nm}$. This degradation of the structural properties can be explained in terms of the growing-layer re-sputtering by the highly energetic impinging species.

Following the obtained results on the effect of $P_{\mathrm{RF}}$, a set of AlN samples were deposited at substrate temperatures ranging from $350{ }^{\circ} \mathrm{C}$ to $500{ }^{\circ} \mathrm{C}$, keeping $P_{\mathrm{RF}}=150 \mathrm{~W}$. For all the samples of this set, only the peak related to the (0002) reflection from AlN was observed in $2 \theta / \omega$ HRXRD measurements, and the RMS surface roughness measured by AFM on $1 \times 1 \mu \mathrm{m}^{2}$ surface remains in the range of $0.3-0.6 \mathrm{~nm}$. As it is shown in Fig. 1(b), a minimum FWHM of the rocking curve of $1.91^{\circ}$ is
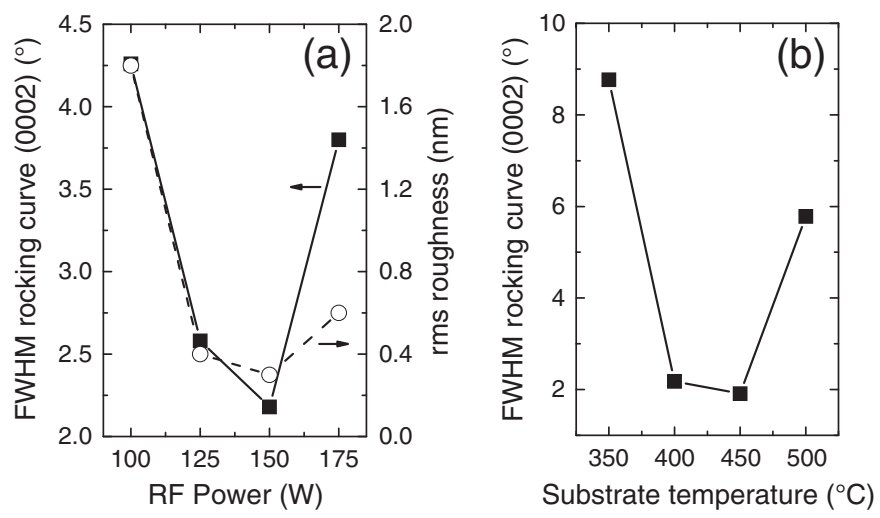

Fig. 1. (a) FWHM of the rocking curve of the AIN-(0002) X-ray reflection and RMS surface roughness of a $1 \times 1 \mu \mathrm{m}^{2}$ scanned area as a function of the RF power for AlN layers deposited on $c$-sapphire at a sputtering pressure of $0.47 \mathrm{~Pa}$ and $T_{\text {subs }}=400{ }^{\circ} \mathrm{C}$. The RMS roughness of the sapphire substrate is $0.2 \mathrm{~nm}$. (b) FWHM of the rocking curve of the AlN-(0002) $\mathrm{X}$-ray reflection as a function of substrate temperature for AlN layers deposited on $c$-sapphire at a sputtering pressure of $0.47 \mathrm{~Pa}$ and $P_{\mathrm{RF}}=150{ }^{\circ} \mathrm{C}$. obtained for $T_{\text {subs }}=450{ }^{\circ} \mathrm{C}$, whereas layers deposited at $350{ }^{\circ} \mathrm{C}$ and $500{ }^{\circ} \mathrm{C}$ show a FWHM of the (0002) rocking curve as high as $8.77^{\circ}$ and $5.78^{\circ}$, respectively. This degradation of the structural quality of the films is attributed to the reduction of the mobility of the impinging species at low substrate temperature and to the increase of the desorption rate of physi-adsorbed atoms at high substrate temperature [20].

The layer deposited at $P_{\mathrm{RF}}=150 \mathrm{~W}$ and $T_{\text {subs }}=450{ }^{\circ} \mathrm{C}$ shows a c-lattice parameter of $4.970 \AA$, which corresponds to a tensile strain of $\varepsilon_{\mathrm{zz}}=0.24 \%$, assuming a relaxed AlN lattice parameter $c_{0}=4.982 \AA$ [22]. The sample presents a layer thickness of $\sim 115 \mathrm{~nm}$ and a grain size of $\sim 40 \mathrm{~nm}$.

\subsection{Effect of the substrate bias}

The adequate tuning of the substrate bias during deposition can be a critical parameter to improve the crystalline quality of the AlN layers [23]. In this section, we describe the effect of substrate bias on the structural quality and surface morphology of AlN layers, using the above-determined deposition conditions (sputtering pressure $=0.47 \mathrm{~Pa}$ in pure nitrogen atmosphere, $P_{\mathrm{RF}}=150 \mathrm{~W}$ and $T_{\text {subs }}=450{ }^{\circ} \mathrm{C}$ ). Considering a substrate floating potential of $-3.8 \mathrm{~V}$ measured during deposition at FS conditions, the substrate bias was varied from $+5 \mathrm{~V}$ to $-35 \mathrm{~V}$ using a DC voltage source connected to the substrate holder through a resistor (56.2 $\Omega$ ). The current $I_{\mathrm{c}}$ flowing in the circuit formed by the polarized substrate, the plasma environment and the sputtering chamber (ground) was monitored during the AlN deposition. The scheme of the experimental system with the electrical connections is shown in Fig. 2 (up). As presented in Fig. 2 (down), the time-dependent evolution of $I_{\mathrm{c}}$ follows the behavior characteristic of a capacitor charging process, with a transient time during which the arrival of positively charged ions partially compensates the externally applied negative bias at the substrate. After this transient time, $I_{\mathrm{c}}$ saturates to a value $I_{s}$ which increases with negative bias.

The variation of substrate bias in the range under study does not have any effect on the growth rate, $60 \pm 5 \mathrm{~nm} / \mathrm{h}$ for all the samples (total growth time $=2 \mathrm{~h}$ ), or on the RMS surface roughness, $0.6 \pm$
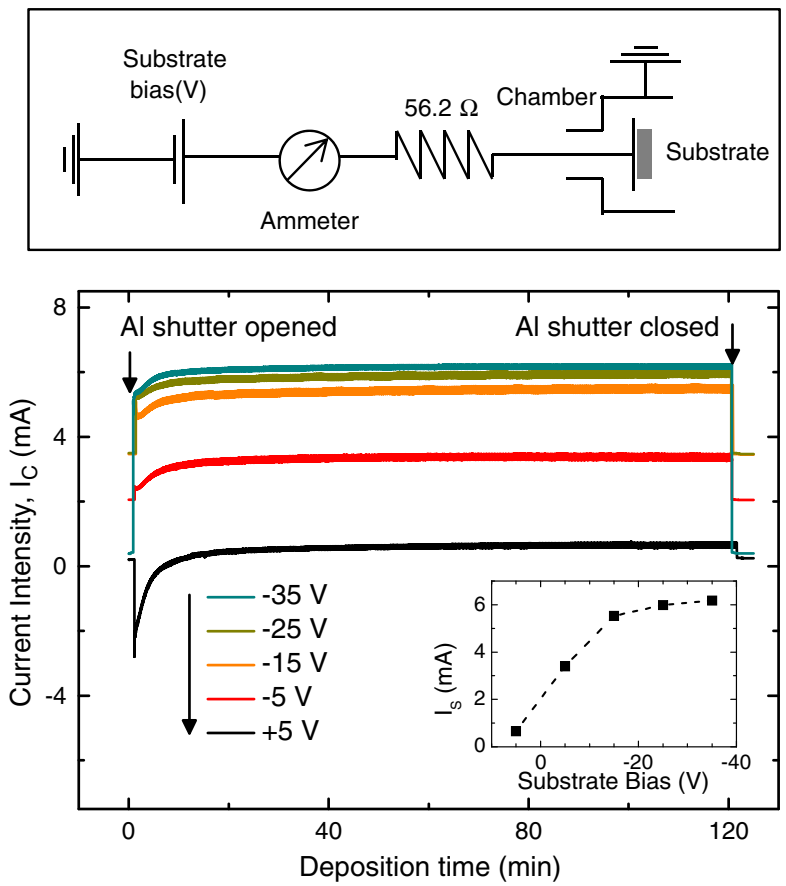

Fig. 2. (Up) Experimental setup used for the process of biasing the substrate during depositions. (Down) Real-time evolution of the current $I_{\mathrm{c}}$ during deposition for different values of substrate bias. Inset: Saturation intensity, $I_{s}$, as a function of the substrate bias. 
$0.1 \mathrm{~nm}$ for $1 \times 1 \mu \mathrm{m}^{2}$ scanning surface. However, differences in the structural quality are observed by HRXRD measurements. Fig. 3 displays the $2 \theta / \omega$ x-ray scans for the set of samples deposited under various substrate bias. The diffractograms show the (0002) AlN peak together with a broad reflection at $2 \theta \sim 35^{\circ}$ for negative bias between $-5 \mathrm{~V}$ and $-25 \mathrm{~V}$. This latter reflection is attributed to lattice disorder at the AlN/sapphire interface originated by the highly accelerated positive ions $\left(\mathrm{Al}^{+}, \mathrm{N}^{+}, \mathrm{N}_{2}^{+}\right)$, impinging the substrate at the initial stages of the deposition process, during the charging transient observed in Fig. 2. In order to verify that the reflection at $2 \theta \sim 35^{\circ}$ is originated by interface damage, we synthesized a sample consisting of a thin ( 30-nm-thick) AlN layer deposited at $-15 \mathrm{~V}$ bias followed by unbiased AlN with a nominal thickness of $240 \mathrm{~nm}$. Indeed, HRXRD measurements from this sample (not shown) presents the AlN-(0002) diffraction peak together with the reflection at $2 \theta \sim 35^{\circ}$, not observed in any unbiased layer, confirming the presence of lattice disorder at the AlN/substrate interface.

Despite the interface damage, the obtained FWHM of the $\omega$-scan of the (0002) AlN diffraction peak decreases monotonously when increasing negative bias up to $-25 \mathrm{~V}$ (see inset of Fig. 3), reaching a best value of $1.25^{\circ}$. The improvement of the structural quality with negative bias can be attributed to an increase of the kinetic energy of the impinging ions, hence increasing the mobility of the adatoms at the growing surface [24]. This is consistent with the observed increase of the grain size, as it is shown in the inset of Fig. 3. A maximum grain size of $\sim 55 \mathrm{~nm}$ is achieved for the AlN sample deposited with a substrate bias of $-15 \mathrm{~V}$.

The AlN film deposited at a substrate bias of $-35 \mathrm{~V}$ shows the highest value of FWHM of the (0002) reflection peak rocking curve in the studied range, namely, $2.23^{\circ}$. The worsening of the structural quality of this sample is attributed to a re-sputtering of the growing surface by the highly accelerated ions [23]. This explanation is supported by the low value of grain size, $\sim 35 \mathrm{~nm}$ in this sample (see inset of Fig. 3). At the same time, the film density estimated by $\mathrm{x}$-ray reflectivity measurements increases from $2.81 \mathrm{~g} / \mathrm{cm}^{3}$ to $3.24 \mathrm{~g} / \mathrm{cm}^{3}$ with the negative substrate bias from $+5 \mathrm{~V}$ to $-15 \mathrm{~V}$. For the sample deposited under $-15 \mathrm{~V}$ bias, the film density is close to the bulk AlN density $\left(3.27 \mathrm{~g} / \mathrm{cm}^{3}\right.$ [25]). The improved compactness of the layers is attributed to the enhancement of the adatom mobility induced by the increased ion kinetic energy [24]. For

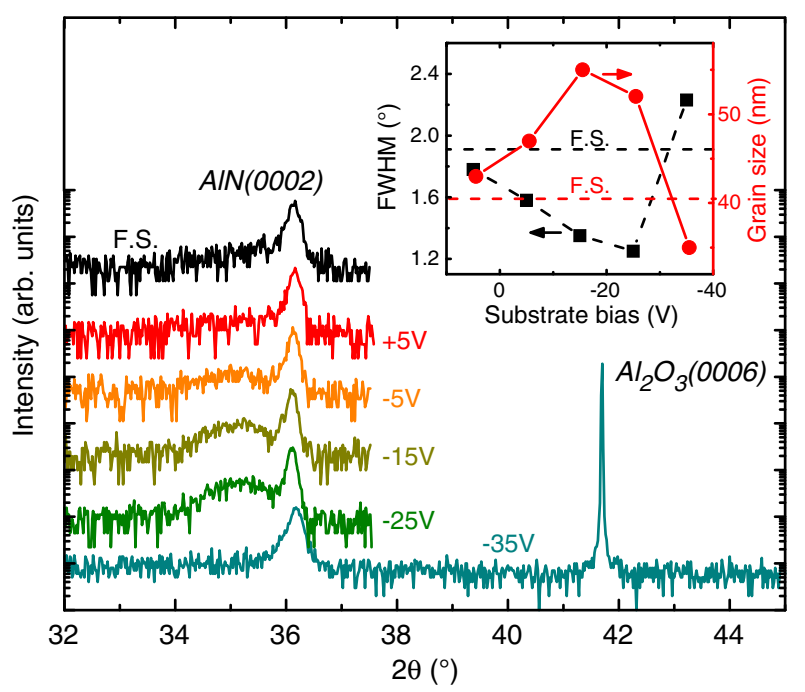

Fig. 3. HRXRD $2 \theta / \omega$-scans of AIN films deposited at $T_{\text {subs }}=450{ }^{\circ} \mathrm{C}$ and $P_{\mathrm{RF}}=150 \mathrm{~W}$ as a function of the substrate bias. The diffractograms are vertically shifted for clarity. Inset: FWHM of the rocking curve of the (0002) reflection and grain size as a function of the substrate bias. Best results obtained for AlN deposited under unbiased conditions (floating substrate, FS) are included as horizontal dashed lines for comparison. substrate bias above this value, the estimated film density decreases to $\sim 2.73 \mathrm{~g} / \mathrm{cm}^{3}$ probably due to a change in layer stoichiometry.

The observed trend with negative bias is somehow similar to the one described as a function of the RF power (see Section 3.1), i.e., increasing $P_{\mathrm{RF}}$ results in a mobility enhancement and thus an improvement of the layer, until a certain threshold $\left(P_{\mathrm{RF}}=175 \mathrm{~W}\right)$ where degradation is associated to the onset of the re-sputtering process.

In summary, within the range of analyzed parameters, the optimized conditions in terms of the FWHM of the (0002) x-ray reflection for AlN growth are $P_{\mathrm{RF}}=150 \mathrm{~W}, T_{\text {subs }}=450{ }^{\circ} \mathrm{C}$, and a substrate bias of $-15 \mathrm{~V}$. For this substrate bias, the AlN layers show the largest grain size $(\sim 55 \mathrm{~nm})$ while keeping low values of FWHM of the $\mathrm{x}$-ray rocking curve $\left(1.35^{\circ}\right)$ and RMS surface roughness $(0.6 \pm 0.1 \mathrm{~nm}$ for a $1 \times 1 \mu \mathrm{m}^{2}$ scan). However, negative bias is associated to a degradation of the substrate/AIN interface. In the next section, we describe an approach to suppress this damage.

\subsection{Two-step deposition method}

A two-step deposition method was studied in biased samples to prevent the lattice disorder observed by HRXRD measurements. This two-step method consists first of the nucleation of an AlN thin layer (buffer layer) under unbiased substrate conditions, followed by the deposition of negatively biased AlN at $-15 \mathrm{~V}$.

The buffer thickness is one of the most important parameters of this two-step growth process. It must be optimized to prevent the interface degradation while keeping it as thin as possible, to profit from the structural improvement under negative bias. Fig. 4 shows the HRXRD $2 \theta / \omega$-scan of two-step samples with unbiased buffer layer thickness of $\sim 15 \mathrm{~nm}$ and $\sim 30 \mathrm{~nm}$, compared to an unbiased layer and a layer grown at $-15 \mathrm{~V}$ bias. The use of an unbiased 15-nm-thick buffer layer does not fully avoid the formation of lattice disorder, noticeable through a weak and broad peak at $2 \theta \sim 35^{\circ}$. This can be understood by the fact that the grain size obtained in thick AlN layers deposited using the same conditions as the buffer layer is $\sim 40 \mathrm{~nm}$, significantly larger than the buffer layer thickness. Thus a non-homogeneous surface coverage can be expected in a three dimensional growth model [26]. The increase of the buffer layer thickness to $30 \mathrm{~nm}$ enables the total suppression of the interface damage (no diffraction peak at $2 \theta \sim 35^{\circ}$ ) and leads to a reduction of the FWHM of the AlN-(0002) reflection peak to $1.63^{\circ}$, to be compared to $\mathrm{FWHM}=1.91^{\circ}$ for the optimized unbiased layer (see

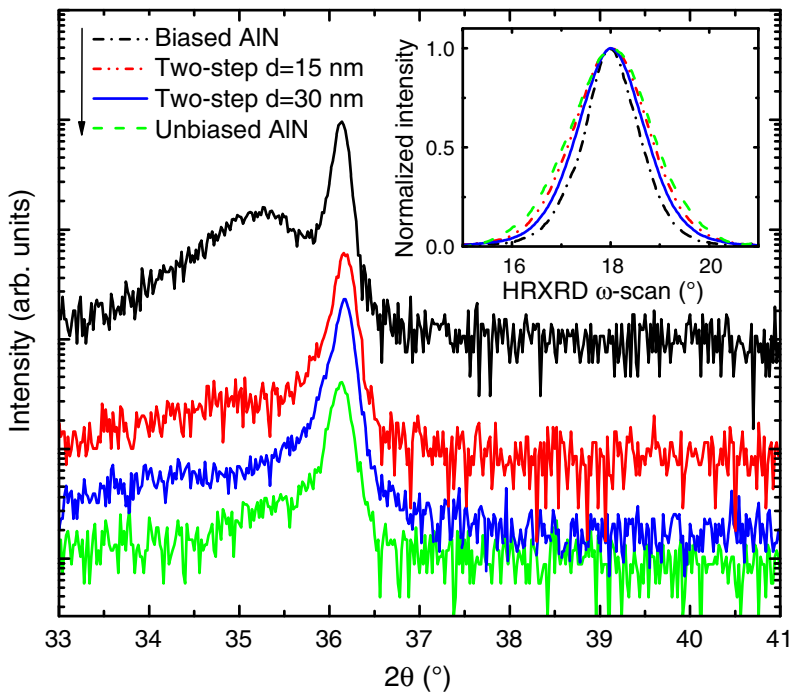

Fig. 4. HRXRD $2 \theta / \omega$-scans for AIN films deposited under various substrate bias conditions: 120 -nm-thick AlN at - $15 \mathrm{~V}$, two-step AlN with 15-nm and 30-nm-thick unbiased buffer layer followed by 120 -nm-thick AIN at $-15 \mathrm{~V}$, and 120 -nm-thick unbiased AlN. The diffractograms are vertically shifted for clarity. 

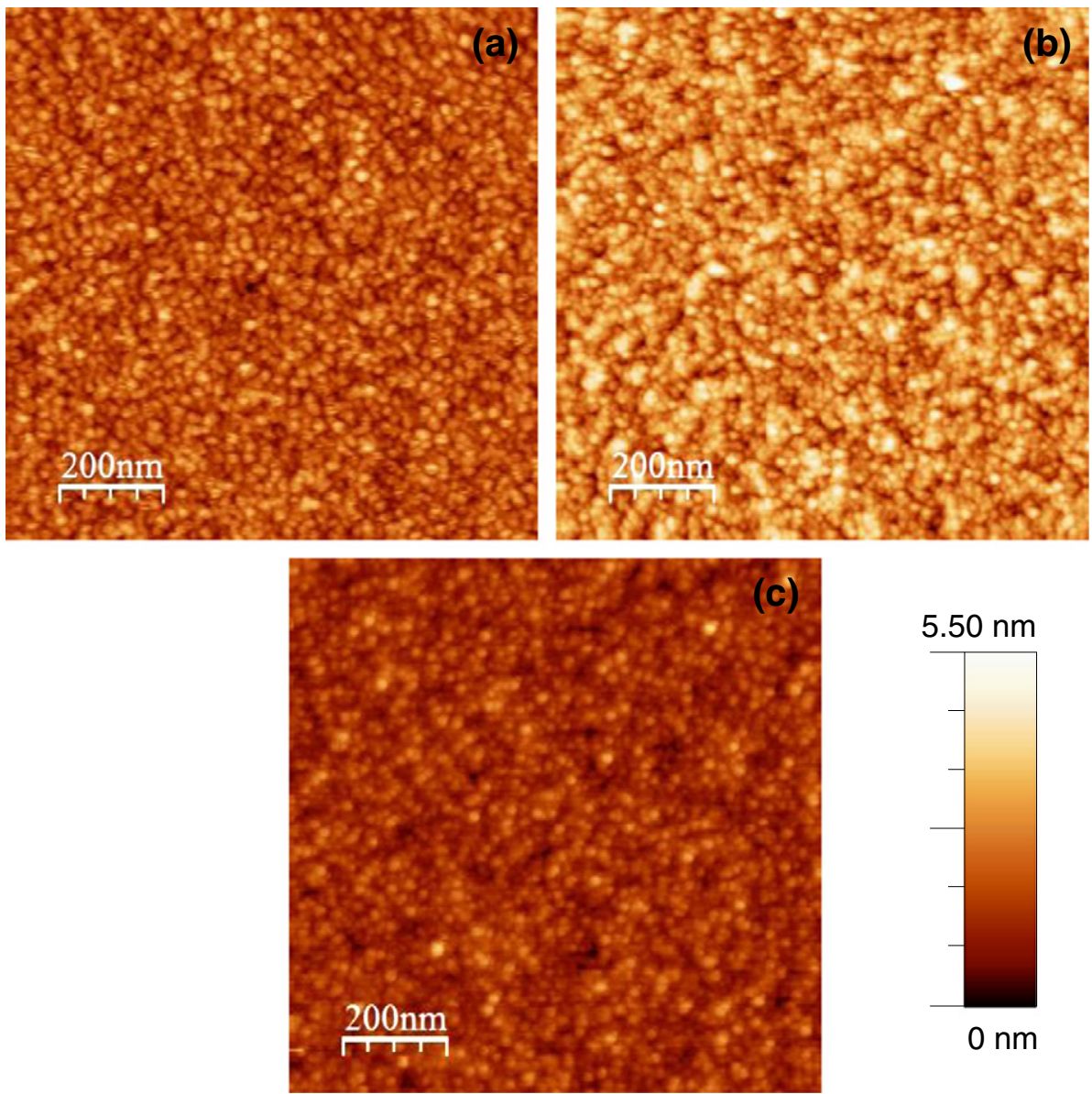

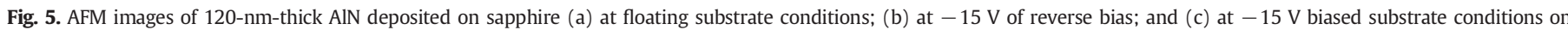
30-nm-thick unbiased AIN buffer layer. The RMS surface roughness of the layers is 0.6, $0.7 \mathrm{~nm}$ and $0.4 \mathrm{~nm}$, respectively.

inset of Fig. 4). The grain size of the 30-nm/120-nm two-step AlN layer is $\sim 40 \mathrm{~nm}$ and the RMS surface roughness is $0.4 \mathrm{~nm}$, measured in a $1 \times 1 \mu \mathrm{m}^{2}$ area. This RMS surface roughness value is lower than the values obtained for both the optimized unbiased and biased AlN layers (see AFM images in Fig. 5).

\section{Conclusions}

The influence of deposition conditions such as RF power, substrate temperature and substrate bias on the morphological and structural properties of $c$-AlN films deposited on $c$-sapphire in pure nitrogen atmosphere by RF reactive sputtering was analyzed. The kinetic energy of impinging ions and thus their mobility at the growing surface is controlled through both the RF power and the substrate bias. The crystalline quality of the layers in terms of grain size and FWHM of the rocking curve is improved when increasing the RF power to an optimal value, established in $150 \mathrm{~W}$ for the employed deposition system. Beyond this threshold, further increase of the RF power becomes deleterious for the layer structural properties due to the onset of the re-sputtering process. The increase of the RF power is accompanied by an enhancement of the growth rate linked to the higher $\mathrm{Al}$ sputtering rate. The use of negative bias to increase the kinetic energy of the plasma species during the deposition allows further improvement of the structural quality. Best results are obtained with bias values between $-15 \mathrm{~V}$ and $-25 \mathrm{~V}$, being this range valid for our system configuration and the substrateholder geometry used in this work. The growth rate does not change with the substrate bias since it increases the kinetic energy of the impinging ions but does not affect to the plasma density, so that the amount of available ions for the deposition remains constant. The improvement of the crystalline quality of the layers under negative substrate bias is accompanied by a degradation of the interface between the AlN layer and the sapphire substrate. This degradation can be avoided by the use of a two-step deposition method consisting of the deposition of an unbiased AlN buffer layer (at least $30 \mathrm{~nm}$ thick) prior to the negatively biased $(-15 \mathrm{~V})$ AlN layer. The optimized AlN film shows a FWHM of the (0002) reflection peak of $1.63^{\circ}$, grain size $\sim 40 \mathrm{~nm}$ and smooth surface morphology with an RMS surface roughness of $0.4 \mathrm{~nm}$.

\section{Acknowledgements}

Partial financial support was provided by the Spanish Government Project, TEC2012-37958-C02-01, and by Comunidad de Madrid Project by No. S2009/ESP-1781. X-ray diffraction measurements were carried out at the X-ray Diffraction Techniques Laboratory at the ICMM-CSIC.

\section{References}

[1] B. Gil, Group III Nitride Semiconductor Compounds, Physics and Applications, Clarendon Press, Oxford, 1998

[2] Y. Taniyasu, M. Kasu, T. Makimoto, Nature 441 (2006) 325

[3] Y. Taniyasu, M. Kasu, Appl. Phys. Lett. 99 (2011) 251112.

[4] M.B. Assouar, M. El Hakiki, O. Elmazria, P. Alnot, C. Tiusan, Diamond Relat. Mater. 13 (2004) 1111.

[5] C. Giordano, I. Ingrosso, M.T. Todaro, G. Maruccio, S. De Guido, R. Cingolani, A Passaseo, M. De Vittorio, Microelectron. Eng. 86 (2009) 1204.

[6] W. Luo, X. Wang, L. Guo, H. Xiao, C. Wang, J. Ran, J. Li, J. Li, Microelectron. J. 39 (2008) 1710 .

[7] J.H. Yang, S.M. Kang, D.V. Dinh, D.H. Yoon, Thin Solid Films 517 (2009) 5057.

[8] C. Yen, W. Lai, Y. Yang, C. Wang, T. Ko, S. Hon, S. Chang, IEEE Photon. Technol. Lett. 24 (2012) 294. 
[9] A.V. Lobanova, E.V. Yakovlev, R.A. Talalaev, S.B. Thapa, F. Scholz, J. Cryst. Growth 310 (2008) 4935.

[10] Y. Taniyasu, M. Kasu, T. Makimoto, J. Cryst. Growth 298 (2007) 315.

[11] D.S. Kamber, Y. Wu, B.A. Haskell, S. Newman, S.P. DenBaars, J.S. Speck, S. Nakamura J. Cryst. Growth 297 (2006) 321.

[12] S. Karmann, H.P.D. Schenk, U. Kaiser, A. Fissel, Wo. Richter, Mater. Sci. Eng. B50 (1997) 228.

[13] G. Koblmüller, R. Averbeck, L. Geelhaar, H. Riechert, W. Hösler, P. Pongratz, J. Appl Phys. 93 (2003) 9591.

[14] Y. Huttel, H. Gomez, A. Cebollada, G. Armelles, M.I. Alonso, J. Cryst. Growth 242 (2002) 116.

[15] Q.X. Guo, T. Tanaka, M. Nishio, H. Ogawa, Vacuum 80 (2006) 716.

[16] S. Valdueza-Felip, F.B. Naranjo, M. González-Herráez, L. Lahourcade, E. Monroy, S. Fernández, J. Cryst. Growth 312 (2010) 2689.
[17] A.L. Patterson, Phys. Rev. Am. Phys. Soc. 56 (1939) 978.

[18] I. Horcas, R. Fernandez, J.M. Gomez-Rodriguez, J. Colchero, J. Gomez-Herrero, A.M. Baro, Rev. Sci. Instrum. 78 (2007) 013705.

[19] Z. Vashaei, T. Aikawa, M. Ohtsuka, H. Kobatake, H. Fukuyama, S. Ikeda, K. Takada, J. Cryst. Growth 311 (2009) 459.

[20] S.D. Ekpe, L.W. Bezuidenhout, S.K. Dew, Thin Solid Films 474 (2005) 330.

[21] A. Palmero, H. Rudolph, F.H.P.M. Habraken, Thin Solid Films 515 (2006) 631.

[22] M.E. Levinshtein, S.L. Rumyantsev, M.S. Shur, Properties of Advanced Semiconductor Materials: GaN, AIN, InN, BN, SiC, SiGe, Wiley, New York, 2001.

[23] M. Clement, E. Iborra, J. Sangrador, A. Sanz-Hervás, L. Vergara, M. Aguilar, J. Appl. Phys. 94 (2003) 1495.

[24] H. Seo, I. Petrov, K. Kim, J. Electron. Mater. 39 (2010) 1146.

[25] T.P. Drüsedau, J. Bläsing, Thin Solid Films 27 (2000) 377.

[26] T.J. Kistenmacher, S.A. Ecelberger, W.A. Bryden, J. Appl. Phys. 74 (1993) 1684 